\title{
A Novel Method Based on Cyclic Spectral Analysis about Carrier Frequency Estimation in the UAV Data Chain
}

\author{
Linxi Zhang ${ }^{1,2, a}$, Peng Song ${ }^{1, b}$, Yingjun Zhang ${ }^{1, c}$ \\ 1. Electronic Information Department, Northwestern Polytechnical University, Xi'an China \\ 2. Science and Technology on UAV Laboratory, Northwestern Polytechnical University, Xi'an China \\ azlx06@nwpu.edu.cn, bsp0188@163.com, cshelter0917@126.com
}

Keywords: low signal-to-noise ratio; cyclic spectral density; BPSK; the time-smoothing FFT accumulation method.

\begin{abstract}
When UAV works on the end of the task path, or affected by waves transmission environment and the influence of the electromagnetic interference, the signal of receiver will be made low signal-to-noise ratio (SNR) signal and the phase lock loop (PLL) using for carrier extraction will performance serious decline or loss locks. Therefore, in order to ensure UAV completed a predefined task successfully, low SNR carrier recovery has become one of the key technologies in the UAV data chain. In this paper, based on the basic concept of cyclic spectral analysis, we research and give the cyclic autocorrelation function and the cyclic spectral density function of the BPSK modulation signal. We emphasis a computer simulation method based on the time-smoothing FFT accumulation method and use this method to verify cyclic spectral analysis is still effective to estimate the carrier frequency when the SNR is $-10 \mathrm{~dB}$.
\end{abstract}

\section{Introduction}

The communication signal of UAV data chain is always a modulated signal, so carrier recovery in most cases has played a very significant role. But when the signal is a low signal-to-noise ratio signal, the phase lock loop for carrier extraction performances serious decline or loss locks. Usually in the loop, $6 \mathrm{~dB}$ SNR of the loop is regarded as the threshold of loss locks [1]. In order to ensure UAV completed a predefined task successfully in the low signal-to-noise ratio circumstances, carrier frequency extraction effectively has become one of the key technologies in the UAV data chain.

Since the development of the cyclic spectral concepts [2,3] by Gardner in the early 1980s, cyclostationary signal processing technique have been extensively used in the signal processing system of communications, radar, and sonar etc., such as spread spectrum communication signal detection and recognition, radar signal detection and parameter estimation, modulation recognition and so on. Cyclostationary signal processing is examining the signal from the time-frequency domain to the frequency-cycle frequency domain. As for white Gaussian noise, its cyclic spectral density is zero at nonzero cycle frequency, in other words, the noise is uncorrelated. Therefore, in order to get rid of noise influence effectively in the parameter estimation, this paper uses cyclostationary signal processing technique to estimate carrier frequency in the low signal-to-noise ratio condition of UAV data chain.

\section{Fundamentals of Cyclostationary}

Generally, the cyclostationary signal is also called periodicity stationary signal, which statistical properties are periodically varying or multi-periodically varying. Now we consider $x(t)$ as a cyclostationary signal and consider $T$ as the length of the time series. The cyclic autocorrelation function is given by [3]

$$
R_{x}^{\alpha}(\tau)=\lim _{T \rightarrow \infty} \frac{1}{T} \int_{-T / 2}^{T / 2} x\left(t+\frac{\tau}{2}\right) x^{*}\left(t-\frac{\tau}{2}\right) e^{-j 2 \pi \alpha t} d t
$$


where $\tau$ is the time interval, and we call $\alpha$ is the cyclic frequency when $R_{x}^{\alpha}(\tau) \neq 0$. From Eq. (1), it is apparent that the cyclic Autocorrelation is actually multiplying autocorrelation function of signal and different coefficient in different cyclic frequency.

As is known that the power spectral density is the Fourier transform of the autocorrelation function, according to this method [4], the spectral correlation density is also obtained from the Fourier transform of the cyclic autocorrelation function as

$$
S_{x}^{\alpha}(f)=\int_{-\infty}^{\infty} R_{x}^{\alpha}(\tau) e^{-j 2 \pi f \tau} d \tau=\lim _{T \rightarrow \infty} \frac{1}{T} X_{T}\left(f+\frac{\alpha}{2}\right) X_{T}^{*}\left(f-\frac{\alpha}{2}\right)
$$

where $\alpha$ is the cycle frequency and $X_{T}(f)$ is the Fourier transform of the time domain signal $x(u)$. It's quite clear that Eqs. 1 and 2 will degenerate for traditional autocorrelation function and power spectral density when $\alpha$ equals zero, accordingly, the additional variable $\alpha$ leads the time-frequency domain to the frequency-cycle frequency domain. Because majority of UAV have adopted BPSK modulation signal, this paper takes BPSK as an example to estimate carrier frequency based on cyclic spectral analysis. The cyclic spectral expressions of BPSK is given by

$$
S_{x}^{\alpha}(f)=\left\{\begin{array}{cc}
\frac{1}{4 T_{c}}\left[Q\left(f-f_{c}+\frac{\alpha}{2}\right) Q^{*}\left(f-f_{c}-\frac{\alpha}{2}\right)+Q\left(f+f_{c}+\frac{\alpha}{2}\right) Q^{*}\left(f+f_{c}-\frac{\alpha}{2}\right)\right], & \alpha=\frac{N}{T_{b}} \\
\frac{1}{4 T_{c}}\left[e^{j 2 \varphi_{0}} Q\left(f-f_{c}+\frac{\alpha}{2}\right) Q^{*}\left(f+f_{c}-\frac{\alpha}{2}\right)+e^{-j 2 \varphi_{c}} Q\left(f+f_{c}+\frac{\alpha}{2}\right) Q^{*}\left(f-f_{c}-\frac{\alpha}{2}\right)\right], & \alpha= \pm 2 f_{c}+\frac{N}{T_{b}} \\
0, & \text { else }
\end{array}\right.
$$

where $Q(f)=\sin \left(\pi f T_{c}\right) / \pi f, \varphi_{0}$ is the initial phase, $T_{b}$ is the chip period and $f_{c}$ is the carrier frequency. In arriving at Eq.3, we can see that the cyclic spectrum of BPSK will appear spectral peak in $\alpha= \pm 2 f_{c}+N / T_{b}$ and $\alpha=N / T_{b}$ (for all integers $\mathrm{N}$ ), among them the spectral peak of $\alpha=0$ and $\alpha= \pm 2 f_{c}$ is the largest.

Estimates of the cyclic spectral density can be obtained via time or frequency-smoothing techniques. Since the signals being analyzed are defined over a finite time interval $\Delta t$, the cyclic spectral density is only an estimate [5]. An estimate of the cyclic spectral density can be obtained by the time-smoothing method given by

$$
S_{x}^{\alpha}(f) \approx S_{x T_{W}}^{\alpha}(t, f)_{\Delta t}=\frac{1}{\Delta t} \int_{t-(\Delta t / 2)}^{t+(\Delta t / 2)} S_{x T_{W}}(u, f) d u
$$

where

$$
S_{x T_{w}}(u, f)=\frac{1}{T_{W}} X_{T_{W}}\left(u, f+\frac{\alpha}{2}\right) X_{T_{W}^{*}}^{*}\left(u, f-\frac{\alpha}{2}\right)
$$

with $\Delta t$ being the total observation time of the signal, $T_{W}$ is the short-time FFT window length, and $X_{T_{W}}(t, f)=\int_{t-\left(T_{W} / 2\right)}^{t+\left(T_{W} / 2\right)} x(u) e^{-j 2 \pi f u} d u$ is the sliding short-time Fourier transform. Figure 1 shows the cyclic spectral density graphically for a signal $x(t)$. 


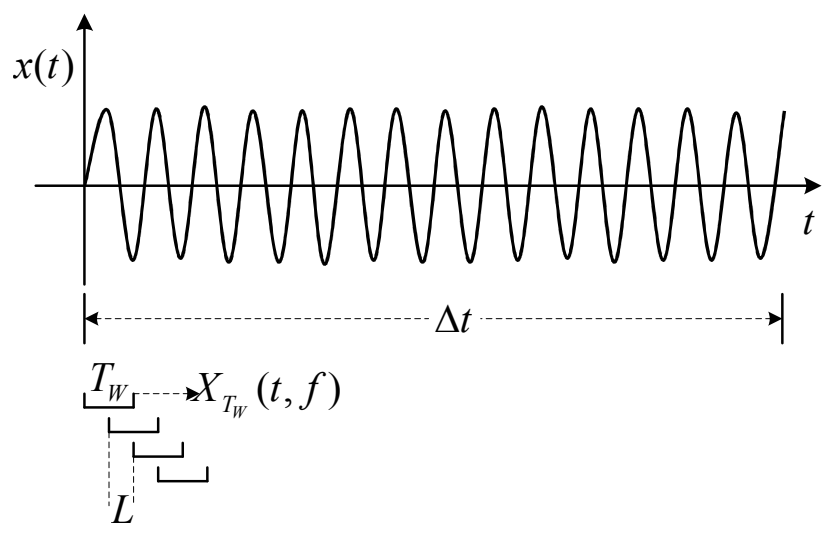

Figure 1: Cyclic spectral density estimation using short-time FFTs.

In the interval $\Delta t$ of signal analysis, the frequency resolution determined by short-time FFT window length, which is $\Delta f=1 / T_{W} . L$ is the overlap factor between each short-time FFT. In order to avoid aliasing and cycle leakage on the estimates, the value $L$ is defined as $L \leq T_{W} / 4$ [6].

In addition, this algorithm must consider a parameter $M=\Delta t / T_{W}$, which name is Grenander's uncertainty condition [2]. In order to obtain a reliable and accurate result, $M$ must be far greater than the value of 1 . It is means that the interval $\Delta t$ must greatly exceed the short-time FFT window length $T_{W}$. The cycle frequency resolution is determined by the interval $\Delta t$, defined as $\Delta \alpha=1 / \Delta t$.

\section{The Time-smoothing FFT Accumulation Method}

The cyclic spectral calculation is due to calculate in frequency-cycle frequency bi-frequency plane, so it is demanding a large amount of calculation. In order to calculate efficiently and realize hardware system, the effective algorithms are indispensable. The time-smoothing FFT accumulation method is an effective algorithm which can reduce the number of computation. Eqs. 4 and 5 can be rewritten to Eqs. 6 and 7 in discrete yields

$$
S_{X_{N^{\prime}}}^{\alpha}(n, f)=\frac{1}{N} \sum_{n=0}^{N-1}\left[\frac{1}{N^{\prime}} X_{N^{\prime}}\left(n, f+\frac{\alpha}{2}\right) X_{N^{\prime}}^{*}\left(n, f-\frac{\alpha}{2}\right)\right]
$$

where

$$
X_{N^{\prime}}(n, f)=\sum_{n=0}^{N^{\prime}-1} w(n) x(n) e^{-(j 2 \pi f n) / N^{\prime}}
$$

is the discrete Fourier transform of $x(n) . w(n)$ is the short-time FFT window length. The parameter $N$ is the total length of data and $N^{\prime}$ is the length of discrete short-time FFT. A block diagram of the time-smoothing FFT accumulation method is shown in Figure 2.

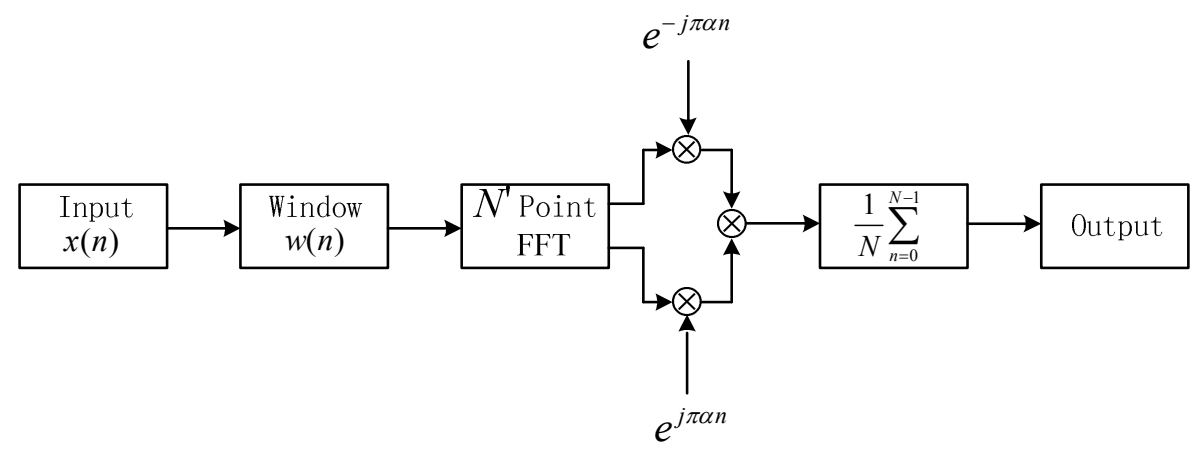

Figure 2: Block diagram of the time-smoothing FFT accumulation method 


\section{Simulation Results}

In this section, we present simulation results by using MATLAB to illustrate the cyclic spectral technique used in estimate of BPSK carrier frequency. Adopted the Time-smoothing FFT Accumulation Method, the test data of signal parameters are the BPSK with a code length of $N=1024$, a chip rate of $R_{b}=20 \mathrm{Mbps}$, a carrier frequency of $f_{c}=2 R_{b}$, a sampling frequency of $f_{s}=4 f_{c}$, a frequency resolution of $\Delta f=f_{s} / 64$, a overlap factor of $L=16$ and a Grenander's uncertainty condition of $M=128$.

In order to prove the cyclic spectral analysis can work more efficiently in low SNR environment, we design the two groups of contrast tests. The first test is an ideal BPSK signal with no noise pollution and the second test is a BPSK signal with white Gaussian noise which SNR is -10dB. The simulation results are shown in Figure 3 and Figure 4.

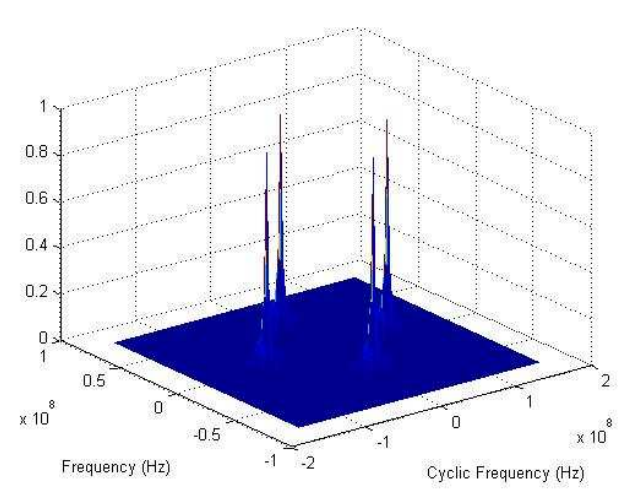

a) bi-frequency plane for no noise signal

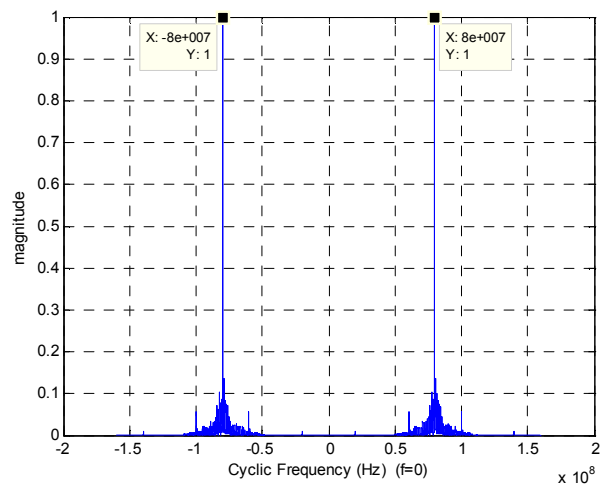

c) slice figure $f=0$ of cyclic frequency

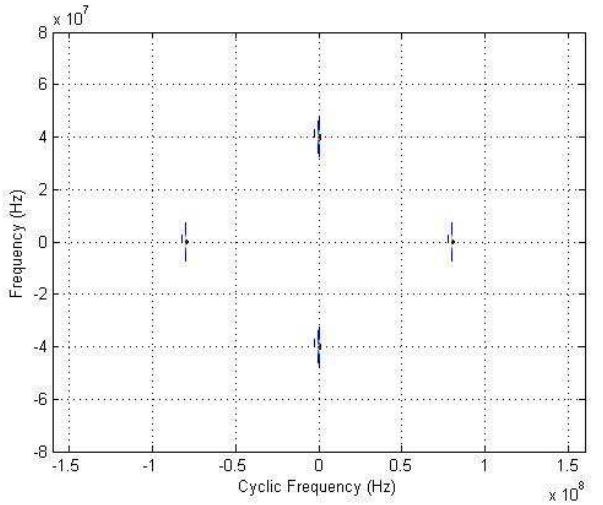

b) skeleton map of bi-frequency plane

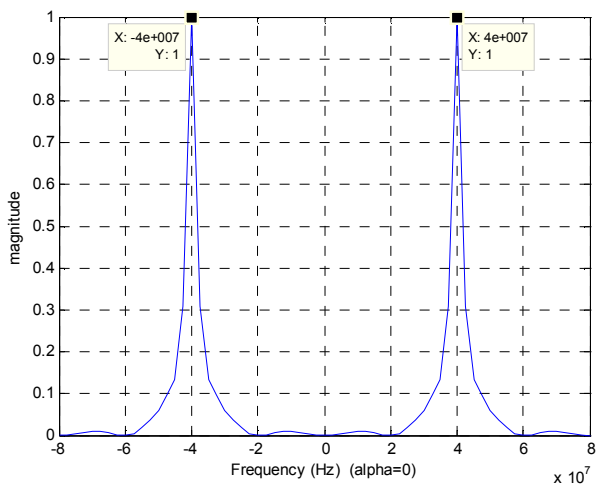

d) slice figure $\alpha=0$ of frequency

Figure 3: Simulation results for no noise signal

The preceding sections described that the cyclic spectrum of BPSK will appear spectral peak in $\alpha= \pm 2 f_{c}+N / T_{b}$ and $\alpha=N / T_{b}$ (for all integers $\mathrm{N}$ ), among them the spectral peak of $\alpha=0$ and $\alpha= \pm 2 f_{c}$ is the largest and the cyclic spectral density function will degenerate for traditional power spectral density function when $\alpha$ equals zero. The Figure 3 a shows that the simulation results have four separate and obvious peaks. To do the slice figure $f=0$ of cyclic frequency, we can see clearly that there are two obvious peaks located in $\alpha= \pm 2 f_{c}$, which equal $8 \times 10^{7} \mathrm{~Hz}$ as shown in Figure 3c. To do the slice figure $\alpha=0$ of frequency, we can see clearly that there are two obvious peaks located in carrier frequency, which is the same as the conventional power spectrum which is shown in Figure 3d. 


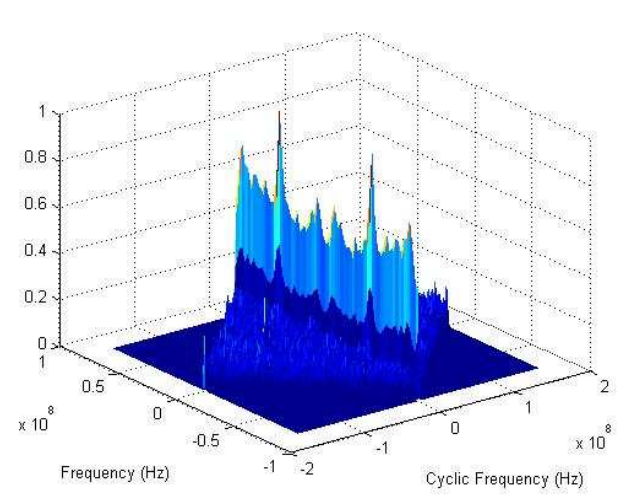

a) bi-frequency plane for $\mathrm{SNR}=-10 \mathrm{~dB}$

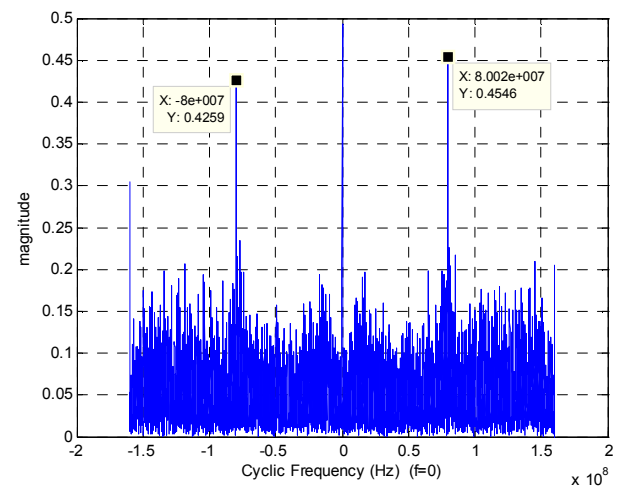

c) slice figure $f=0$ of cyclic frequency

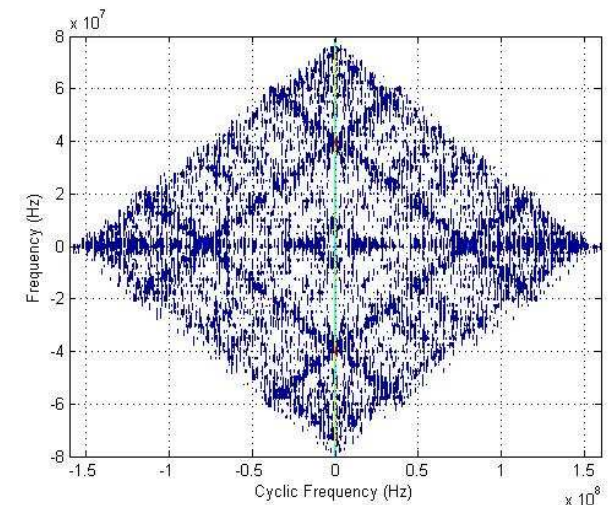

b) skeleton map of bi-frequency plane

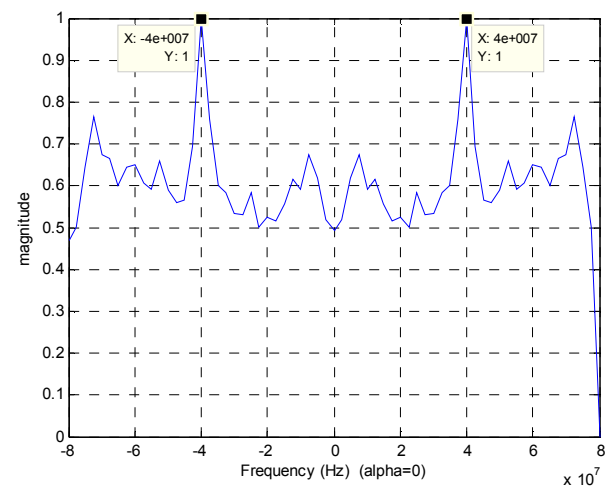

d) slice figure $\alpha=0$ of frequency

Figure 4: Simulation results for $\mathrm{SNR}=-10 \mathrm{~dB}$

When the SNR is deteriorated to $-10 \mathrm{~dB}$, as shown in figure $4 \mathrm{c}$ of slice figure $f=0$ of cyclic frequency, we have also discovered two obvious distinct peaks existed in the positive frequency $\alpha=8.002 \times 10^{7}$ and negative frequency $\alpha=-8 \times 10^{7}$, which is very close to the theoretical value.

\section{Summary}

This paper introduces the basic theories of the cyclic spectral density and realizes the computer simulations of BPSK signal by using the time-smoothing FFT accumulation method. The simulation results have proved that in low SNR, cyclic spectral analysis can still identify the carrier frequency effectively. Therefore, cyclic spectral technology will become a novel and effective method in estimating carrier frequency of low SNR signal at UAV data chain.

\section{Acknowledgements}

This research was supported by the Research Foundation of the Pre-assembly under Grant 9140A25030511HK03.

\section{Reference}

[1] Yang Yonghui. "Analysis on the Pulse Signal Jamming the Carrier Extracted Phase-locked Loop," Communications Technology, 2007: No.05.

[2] W. A. Gardner. "Measurement of spectral correlation," IEEE Trans. ASSP, 1986, ASSP-34(10):1111-1123.

[3] W. A. Gardner. "The spectral correlation theory of cyclostationary time-series," Signal Processing [j], 1986, 11(1):13-36. 
[4] W. A. Gardner., W. A. Brown, C. K. Chen. "Spectral correlation of modulated signal: part1-analog modulation," IEEE Transactions on Communication, 1987, 35(6):584-594.

[5] Phillip E P. Detecting and classifying low probability of intercept radar second edition. Artech House, 2009, pp. 513-530.

[6] Lima, A. F., Jr., "Analysis of low probability of intercept radar signals using cyclostationary processing,” Naval Postgraduate School Master's thesis, Sept. 2002. 